\title{
On the Kernel of the Klein-Gordon Operator
}

\author{
V. V. Kravchenko and R. Castillo P.
}

\begin{abstract}
In this work with the aid of technics of quaternionic analysis we show that any solution of the Klein-Gordon equation can be represented via two solutions of the Dirac equation with the same mass. Moreover, the two functions corresponding to each solution of the KleinGordon equation are unique.
\end{abstract}

Keywords: Klein-Gordon operator, Dirac operator, quaternions

AMS subject classiflcation: 81 Q 05

\section{Introduction}

In this work we study the relationship between two most important operators of particle physics, namely the Klein-Gordon operator and the Dirac operator. Of course, some relations between them were established in the moment when Dirac discovered his operator as a square root of the Klein-Gordon operator. Thus, it is clear that any solution of the Dirac equation solves also the Klein-Gordon equation. Here using the formalism and methods of quaternionic analysis we prove a more intimate connection between the solutions of both equations. Namely, we show that any solution of the Klein-Gordon equation can be represented via two solutions of the Dirac equation with the same mass that seems to be a natural extension of Dirac's theory. In other words, any function describing the behaviour of a free particle with integer spin can be completely determined by two functions describing free particles with spin $\frac{1}{2}$. Moreover, the two functions corresponding to each solution of the Klein-Gordon equation are unique.

The methods of quaternionic analysis used in this article were developed recently in $[1,4,8]$. Here in Section 2 we present only some necessary definitions and results referring the reader to corresponding works. Section 3 contains the main results of the article resumed in Proposition 3.

V. V. Kravchenko: Escuela Superior de Ingeniería Mecánica y Eléctrica, Instituto Politécnico Nacional, Unidad-Zacatenco, C.P. 07738 Mexico-City, México

R. Castillo P.: Escuela Superior de Ingeniería Mecánica y Eléctrica, Instituto Politécnico Nacional, Unidad-Zacatenco, C.P. 07738 Mexico-City, México 


\section{Preliminaries}

We denote by $\mathbb{H}(\mathbb{C})$ the algebra of complex quaternions (i.e. biquaternions). Each element $\alpha \in \mathbb{H}(\mathbb{C})$ is represented in the form $\alpha=\sum_{k=0}^{3} \alpha_{k} i_{k}$, where $\alpha_{k} \in \mathbb{C}, i_{0}$ is the unit and $i_{k}$ are standard basic quaternions. We denote the imaginary unit in $\mathbb{C}$ by $i$ as usual. By definition $i$ commutes with all $i_{k}$. The quaternion $\bar{\alpha}=\alpha_{0}-\sum_{k=1}^{3} \alpha_{k} i_{k}$ is called conjugated to $\alpha$. For multiplication from the right-hand side by a quaternion $\alpha$ we use the notation $M^{\alpha}$, i.e. by definition $M^{\alpha} f=f \alpha$.

Let us denote by $\mathfrak{S}$ the set of zero divisors from $\mathbb{H}(\mathbb{C})$. This is the set of all complex quaternions $\alpha$ satisfying the condition $\alpha \bar{\alpha}=0$. As usual zero is not included into $\mathfrak{S}$. We will use the following idempotents generated by zero divisors:

$$
P_{k}^{ \pm}=\frac{1}{2} M^{\left(1 \pm i i_{k}\right)} \quad(1 \leq k \leq 3) .
$$

Let us consider the Helmholtz operator with quaternionic wave number $\Delta+M^{\alpha^{2}}$ studied in [7] (see also [8]). Here $\Delta$ is the 3-dimensional Laplace operator. With the aid of quaternions it is possible (see [3]) to factorize the Helmholtz operator:

$$
\Delta+M^{\alpha^{2}}=-\left(D+M^{\alpha}\right)\left(D-M^{\alpha}\right),
$$

where $D=\sum_{k=1}^{3} i_{k} \frac{\partial}{\partial x_{k}}$ is the well-known Moisil-Theodoresco operator, which was studied for the first time in $[9,10]$. Moreover, for $\alpha \notin \mathfrak{S} \cup\{0\}$,

$$
\operatorname{ker}\left(\Delta+M^{\alpha^{2}}\right)=\operatorname{ker}\left(D+M^{\alpha}\right) \oplus \operatorname{ker}\left(D-M^{\alpha}\right) \text {. }
$$

For $\alpha \in \mathfrak{S}$ we have

$$
\operatorname{ker}\left(\Delta+M^{\alpha^{2}}\right)=M^{\alpha}\left(\operatorname{ker} D_{2 \alpha_{0}} \oplus \operatorname{ker} D_{-2 \alpha_{0}}\right) \oplus M^{\bar{\alpha}}(\operatorname{ker} \Delta) .
$$

Here and in what follows we use the notation $D_{\alpha}=D+M^{\alpha}$. In its turn for the kernel of the Laplace operator we have

$$
\operatorname{ker} \Delta=\operatorname{ker} D_{-\alpha} \oplus M^{i_{1}}\left(\operatorname{ker} D_{\alpha}\right)
$$

where $\alpha$ is an arbitrary zero divisor, $\alpha_{0}=0$, and $\alpha_{1} \neq 0$. These results were obtained in $[6,7]$ (see also [8: Section 2]) with the help of the operators

$$
\Pi_{\alpha}= \begin{cases}-\frac{1}{2 \alpha \bar{\alpha}} M^{\bar{\alpha}} D_{-\alpha} & \text { if } \alpha \notin \mathfrak{S} \\ -\frac{1}{8 \alpha_{0}^{2}} M^{\alpha} D_{-\alpha} & \text { if } \alpha \in \mathfrak{S}\end{cases}
$$

which are defined on $\operatorname{ker}\left(\Delta+M^{\alpha^{2}}\right)$ and possess the following properties:

$$
\begin{aligned}
\Pi_{\alpha}^{2} & =\Pi_{\alpha} & & \text { for all } \alpha \in \mathbb{H}(\mathbb{C}) \\
\Pi_{\alpha} \Pi_{-\alpha}=\Pi_{-\alpha} \Pi_{\alpha} & =0 & & \text { for all } \alpha \in \mathbb{H}(\mathbb{C}) \\
\Pi_{\alpha}+\Pi_{-\alpha} & =I & & \text { for all } \alpha \notin \mathfrak{S} \\
\Pi_{\alpha}+\Pi_{-\alpha}+\frac{1}{2 \alpha_{0}} M^{\bar{\alpha}} & =I & & \text { for all } \alpha \in \mathfrak{S} .
\end{aligned}
$$


For any $\alpha \in \mathbb{H}(\mathbb{C})$ we have $\Pi_{\alpha}: \operatorname{ker}\left(\Delta+M^{\alpha^{2}}\right) \longrightarrow \operatorname{ker} D_{\alpha}$.

We will consider $\mathbb{H}(\mathbb{C})$-valued functions given in a domain $\Omega \subset \mathbb{R}^{4}$. On the set $C^{1}(\Omega ; \mathbb{H}(\mathbb{C}))$ the following operator is defined $[5]$ :

$$
\mathbb{D}=P_{1}^{+}\left(i \partial_{t}+D\right)+P_{1}^{-}\left(-i \partial_{t}+D\right)-m M^{i_{2}},
$$

where $\partial_{t}=\frac{\partial}{\partial t}$. The operator $\mathbb{D}$ is equivalent to the classic Dirac operator

$$
\mathfrak{D}=\gamma_{0} \partial_{t}-\sum_{k=1}^{3} \gamma_{k} \frac{\partial}{\partial x_{k}}+i m I
$$

in the sense that $\mathbb{D}$ may be obtained from $\mathfrak{D}$ by a simple invertible matrix transformation (see [5] and [8: Section 12]). We denote by $F$ the Fourier transform with respect to the variable $t$ :

$$
F[f(t)]=\int_{-\infty}^{\infty} f(t) e^{-i \beta t} d t
$$

\section{Decomposition of the kernel of the Klein-Gordon operator}

Let us denote by $A$ the Klein-Gordon operator which describes free particles with integer spin:

$$
A=\partial_{t}^{2}-\Delta+m^{2} \text {. }
$$

We will consider the operator $A$ on the set $C^{2}(\Omega)$, where $\Omega=(-\infty, \infty) \times G,(-\infty, \infty)$ is the axis corresponding to the time variable $t$ and $G$ is a domain in $\mathbb{R}^{3}$. Let us introduce the operator

$$
\overline{\mathbb{D}}=P_{1}{ }^{+}\left(-i \partial_{t}+D\right)+P_{1}{ }^{-}\left(i \partial_{t}+D\right)+m M^{i_{2}}
$$

and consider the product

$$
\mathbb{D} \overline{\mathbb{D}}=P_{1}{ }^{+}\left(\partial_{t}{ }^{2}-\Delta\right)+P_{1}{ }^{-}\left(\partial_{t}{ }^{2}-\Delta\right)+m^{2}=\partial_{t}{ }^{2}-\Delta+m^{2}=A .
$$

Thus, $A=\mathbb{D} \overline{\mathbb{D}}$. We will use this fact for analysis of $\operatorname{ker} A$.

Let us rewrite the operators $A, \mathbb{D}$ and $\overline{\mathbb{D}}$ in the form

$$
\begin{aligned}
& A=F^{-1}\left(-\Delta+m^{2}-\beta^{2}\right) F \\
& \mathbb{D}=F^{-1}\left(D+M^{\left(i \beta i_{1}-m i_{2}\right)}\right) F \\
& \overline{\mathbb{D}}=F^{-1}\left(D-M^{\left(i \beta i_{1}-m i_{2}\right)}\right) F .
\end{aligned}
$$

Let us denote $\alpha=-\left(i \beta i_{1}-m i_{2}\right)$. Then for the Helmholtz operator from the brackets in (7) we have

$$
-\Delta+m^{2}-\beta^{2}=D_{\alpha} D_{-\alpha} .
$$

Using the corresponding projection operators $\Pi_{\alpha}$ and $\Pi_{-\alpha}$ we introduce the operators

$$
Q_{ \pm \alpha}=F^{-1} \Pi_{ \pm \alpha} F
$$

The following proposition is a simple corrollary of (3) - (6). 
Proposition 1. On the set $\operatorname{ker} A$ the following equalities are true:

1. $Q_{\alpha}^{2}=Q_{\alpha}$.

2. $Q_{\alpha} Q_{-\alpha}=Q_{-\alpha} Q_{\alpha}=0$.

3. $Q_{\alpha}+Q_{-\alpha}=I$.

Note that $\operatorname{ker} Q_{\alpha}=\operatorname{ker} \mathbb{D}$ and $\operatorname{ker} Q_{-\alpha}=\operatorname{ker} \overline{\mathbb{D}}$. The prove, for example, of the first equality is a corrollary of the fact that both inclusions $f \in \operatorname{ker} Q_{\alpha}$ and $f \in \operatorname{ker} \mathbb{D}$ are equivalent to the equality $D_{-\alpha} F f=0$. Then we obtain the following

Proposition 2. The kernel of the Klein-Gordon operator $A$ can be represented as direct sum of the kernels of Dirac's operators $\mathbb{D}$ and $\overline{\mathbb{D}}$, i.e. $\operatorname{ker} A=\operatorname{ker} \mathbb{D} \oplus \operatorname{ker} \overline{\mathbb{D}}$.

This proposition is a generalization of the following result obtained in [2]: $\mathcal{M}=$ $\operatorname{ker}\left(-i \partial_{t}+D\right) \oplus \operatorname{ker}\left(i \partial_{t}+D\right)$, where $\mathcal{M}$ denotes all null-solutions of the wave operator $\Delta-\partial_{t}^{2}$ which depend on time.

Note that if $f \in \operatorname{ker} \mathbb{D}$, then $f i_{3} \in \operatorname{ker} \overline{\mathbb{D}}$. In other words: $\operatorname{ker} \overline{\mathbb{D}}=M^{i_{3}}(\operatorname{ker} \mathbb{D})$ and we obtain the following principal result of this article:

Proposition 3. The relation $\operatorname{ker} A=\operatorname{ker} \mathbb{D} \oplus M^{i_{3}}(\operatorname{ker} \mathbb{D})$ is true.

A curious point in this result is the fact that due to the anticommutativity of the imaginary quaternionic units the kernel of the operator of second order $A$ is represented via the kernel of the operator of first order $\mathbb{D}$. Consequently, any solution of the KleinGordon equation may be represented as a sum of two solutions of the Dirac equation one of which is multiplied by $i_{3}$. This result conforms, e.g., with the quark model of mesons. Each meson is constituted by one quark and one anti-quark. The multiplication by $i_{3}$ in Proposition 3 represents the transformation of a particle into an anti-particle.

Note that decomposition (1) of the kernel of Helmholtz's operator is not unique and can be rewritten in a form similar to Proposition 3. Let us consider the following Helmholtz operator $\Delta+\alpha^{2} I$, where $\alpha \in \mathbb{C}$. Then we have

$$
\Delta+\alpha^{2} I=-\left(D+M^{\alpha i_{1}}\right)\left(D-M^{\alpha i_{1}}\right)
$$

and with the aid of the projection operators $\Pi_{\alpha}$ and $\Pi_{-\alpha}$ we obtain

$$
\operatorname{ker}\left(\Delta+\alpha^{2} I\right)=\operatorname{ker}\left(D+M^{\alpha i_{1}}\right) \oplus \operatorname{ker}\left(D-M^{\alpha i_{1}}\right) .
$$

But $\operatorname{ker}\left(D-M^{\alpha i_{1}}\right)=M^{i_{2}}\left(\operatorname{ker}\left(D+M^{\alpha i_{1}}\right)\right)$, consequently

$$
\operatorname{ker}\left(\Delta+\alpha^{2} I\right)=\operatorname{ker}\left(D+M^{\alpha i_{1}}\right) \oplus M^{i_{2}}\left(\operatorname{ker}\left(D+M^{\alpha i_{1}}\right)\right) .
$$




\section{References}

[1] Berezin, A. V., Kurochkin, Yu. A. and E. A. Tolkachev: Quaternions in Relativistic Physics (in Russian). Minsk: Nauka y Tekhnika 1989.

[2] Castillo, R. and V. V. Kravchenko: Sobre una descomposición del kernel del operador de onda. In: Memorias del 3er Congreso de Proyectos de Investigación, México D.F. 1997 (ed.: A. Vázquez M.). Mexico City: Nat. Politechn. Inst. 1997, pp. 1 - 5.

[3] Gürlebeck, K.: Hypercomplex factorization of the Helmholtz equation. Z. Anal. Anw. 5 (1986), $125-131$.

[4] Gürlebeck, K. and W. Sprößig: Quaternionic Analysis and Elliptic Boundary Value Problems. Berlin: Akademie-Verlag 1989.

[5] Kravchenko, V. V.: On a biquaternionic bag model. Z. Anal. Anw. 14 (1995), 3 - 14.

[6] Kravchenko, V. V.:- Direct sum expansion of the kernel of the Laplace operator with the aid of biquaternion zero divisors. Diff. Equ. 31 (1995), $462-465$.

[7] Kravchenko, V. V. and M. V. Shapiro: Helmholtz operator with a quatemionic wave number and associated function theory. In: Deformations of Mathematical Structures, Vol. II (ed.: J. Lawrynovicz). Dordrecht: Kluwer Acad. Publ. 1994, pp. $101-128$.

[8] Kravchenko, V. V. and M. V. Shapiro: Integral Representations for Spatial Models of Mathematical Physics (Pitman Res. Notes Math. Ser.: Vol. 351). Harlow: Longman 1996.

[9] Moisil, G.: Sur les quaternions monogenes. Bull. Sci. Math. Paris 55 (1931), $169-194$.

[10] Moisil, G. and N. Theodoresco: Functions holomorphes dans l'espace. Mathematica (Cluj) 5 (1931), $142-159$. 